\title{
ESTUDO DO PROCESSO DE TREFILAÇÃO DE FIOS DE COBRE ATRAVÉS DE UMA MÁQUINẢ DE TRAÇÃO *
}

\author{
Willy Ank de Morais ${ }^{1}$ \\ Antônio Felipe Costa Sulino ${ }^{2}$ \\ Christopher Adolfo ${ }^{3}$ \\ Igor Santos Paulo ${ }^{4}$ \\ Nohan Ramos Oliveira de Morais ${ }^{5}$
}

\section{Resumo}

Com o objetivo de simular o processo de trefilação, através dos recursos disponibilizados por uma máquina de tração, foram realizadas simulações do processo de conformação do fio de cobre. Utilizando a máquina de tração do laboratório de metrologia da UNISANTA, foram obtidos parâmetros afim que permitem avaliar o processo de trefilação. Os parâmetros obtidos, a eficiência do processo, as propriedades do material e outras informações foram avaliadas através de métodos analíticos.

Palavras-chave: Trefilação; Simulação; Máquina de Tração; Conformação.

\section{STUDY OF WIRES DRAWING PROCESS BY THE USE OF A TENSILE TEST MACHINE}

\begin{abstract}
In order to simulate the drawing process, through the resources available by a tensile test machine, forming process simulations of copper wire were performed. Using the tensile test machine of the metrology laboratory at UNISANTA were obtained parameters in order for evaluating the drawing process. The obtained parameters, process efficiency, the material properties and other information were assessed by analytical methods.
\end{abstract}

Keywords: Drawing; Simulation; Tensile Machine; Forming.

1 Doutorando, Mestre, Engenheiro e Técnico em Metalurgia e Materiais, Sócio-Diretor da Willy Ank Soluções Metal-Mecânicas, Professor Mestre da UNAERP-Guarujá e Professor Adjunto da Universidade Santa Cecília (UNISANTA), Santos, SP, Brasil (wmorais@unisanta.br).

2 Graduando em Engenharia Mecânica, UNISANTA, Santos, SP, Brasil (felipe_sulino@hotmail.com).

3 Graduando em Engenharia Mecânica, UNISANTA, Santos, SP, Brasil (adolfochristo@gmail.com).

4 Graduando em Engenharia Mecânica, UNISANTA, Santos, SP, Brasil (igor_paulo93@hotmail.com).

5 Graduando em Engenharia Mecânica, UNISANTA, Santos, SP, Brasil (nohanmorais@gmail.com). 


\section{INTRODUÇÃO}

A trefilação é o processo mecânico de conformação plástica dos metais, esse processo acarreta na redução da largura do material, consequentemente aumentando o seu comprimento. $\mathrm{O}$ processo consiste em forçar a passagem do material por uma matriz ao mesmo tempo em que tracionado, desta maneira se consegue pequenas espessuras, como por exemplo corda de instrumentos musicais.

No presente trabalho uma máquina de tração da UNISANTA, que fora adequada para executar simulações do processo de trefilação de fios [1], foi empregada para avaliar condições variadas do processo de trefilação de fios de cobre.

Os parâmetros obtidos do material servem como dados de entrada de métodos analíticos teóricos, tipicamente empregados, conforme descritos na literatura [2], para analisar o processo de trefilação. Empregando-se os dados gerados nos ensaios de caracterização do material e os dados obtidos durante as simulações, foi possível comparar matematicamente os resultados práticos obtidos com os valores teóricos calculados.

\section{MATERIAIS E MÉTODOS}

\subsection{Montagem Experimental}

Empregou-se um porta fieira e a montagem de simulação da trefilação em máquina de tração de acordo com o trabalho desenvolvido por Morais et. al. [1]. A Figura 1 ilustra algumas das características da montagem empregada. A fieira empregada (Fig. 1.c) foi usinada na própria universidade e é constituída de aço SAE J404 4043 temperada em óleo. O dispositivo de fixação de fieira (porta fieira, Fig. 1.b) foi projetando e construindo a partir de um tubo SCH 60 com diâmetro de 11/4 pol. e comprimento de $80 \mathrm{~mm}$. A este tubo foi soldado um tarugo de aço carbono de diâmetro de $42 \mathrm{~mm}$ de forma a permitir o encaixe do conjunto na garra superior da máquina de tração (Fig. 1.a).
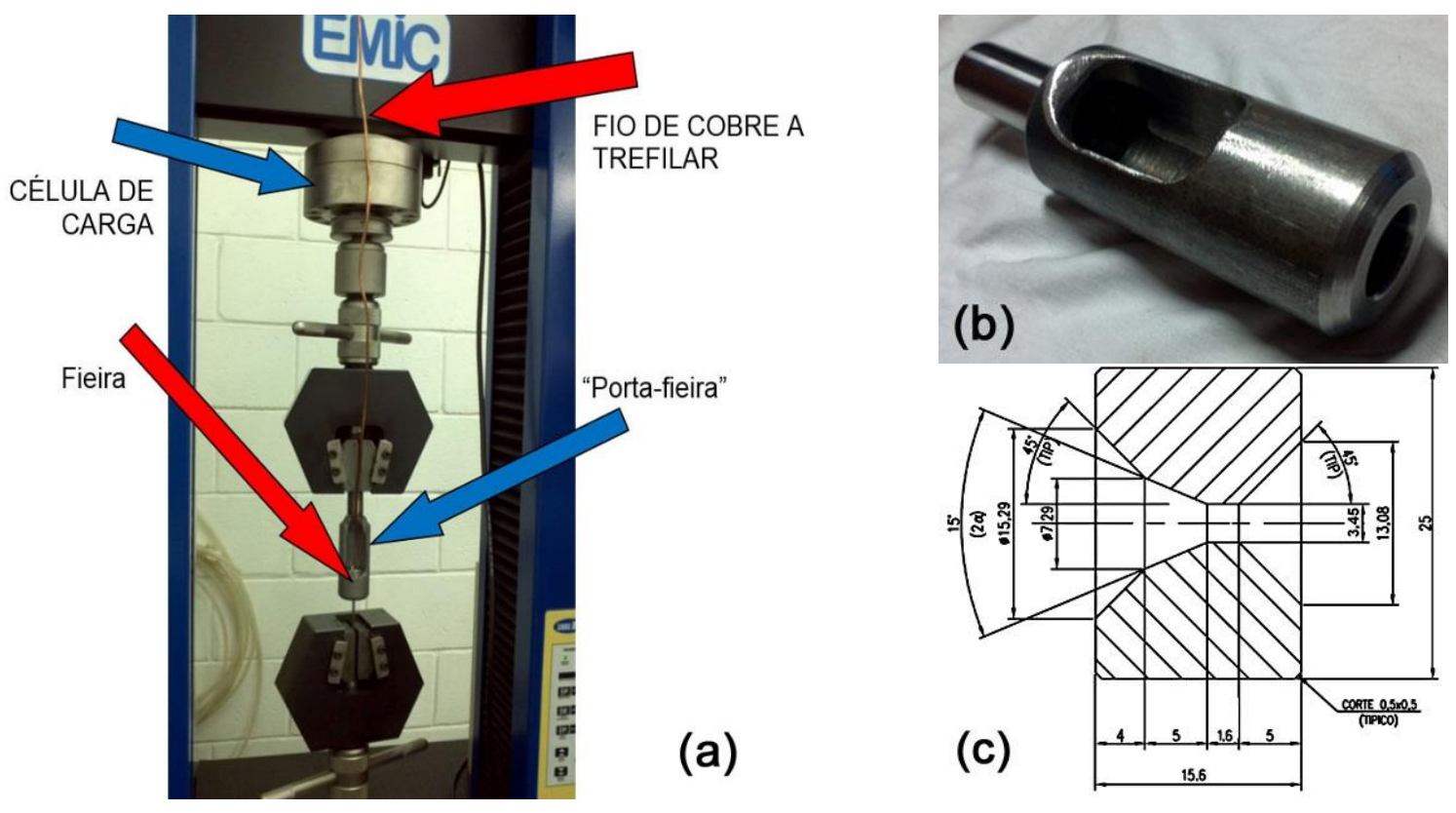
Figura 1. Montagem experimental empregada: (a) porta fieira; (b) dimensões da fieira e (c) conjunto montado na máquina de tração. Fonte: Morais et. al. [1].

\subsection{Condições de Simulação da Trefilação}

Para realizar a trefilação foi utilizado um fio de cobre com diâmetro de $3,68 \mathrm{~mm}$, empregado como condutor elétrico. Após desencapado, o fio passou por um recozimento no forno do laboratório metalográfico da UNISANTA a $500^{\circ} \mathrm{C}$, para minimizar os vestígios de encruamento no seu manuseio e homogeneizar a amostra. O processo de trefilação ocorreu com uma redução de $6,250 \%$ do diâmetro (de $\mathrm{D}_{0}=$ $3,68 \mathrm{~mm}$ para $D_{f}=3,45 \mathrm{~mm}$ ) ou de $12,11 \%$ em área (de $10,6 \mathrm{~mm}^{2}$ para $9,35 \mathrm{~mm}^{2}$ ). Foram utilizados CPs de $2 \mathrm{~m}$ de comprimento, os quais foram trefilados a uma distância de $200 \mathrm{~mm}$. Todos os materiais trefilados foram previamente recozidos e cada CPs teve o seu diâmetro medido em 3 pontos diferentes. A seguir, os CPs empregados para simular o processo de trefilação foram conformados na máquina de tração conforme as condições descritas na Tabela 1.

\begin{tabular}{ccc|}
$\begin{array}{c}\text { Tabela 1. Condições de trefilação empregadas. } \\
\begin{array}{c}\text { Quantidade de } \\
\text { CPs }\end{array}\end{array}$ & $\begin{array}{c}\text { Condição de } \\
\text { Lubrificação }\end{array}$ & $\begin{array}{c}\text { Velocidade } \\
\text { (mm/min.) }\end{array}$ \\
\hline 5 & Sem lubrificação & 50 \\
\hline 2 & Grafite em pó & 200 \\
\hline 3 & Óleo & 50 \\
\hline 3 & & 200 \\
\hline 2 & & 50 \\
\hline
\end{tabular}

\subsection{Caracterização via Ensaio de Tração}

Com a utilização de um extensômetro foram obtidos valores precisos de deformação para cada corpo de prova, antes e depois de trefilado no sistema (Fig. 1.C). A partir da determinação da tensão de engenharia $\left(S=F_{i} / A_{0}\right)$ e da deformação de engenharia $\left(\mathrm{e}=\Delta \mathrm{L} / \mathrm{L}_{0}\right)$ é possível determinar o valor da tensão real $\left(\sigma=F_{i} / A_{i}\right)$ e da deformação real $\left(\mathcal{E}=\ln \left(L_{i} / L_{0}\right)\right)$, levando em consideração um processo de deformação plástica homogênea:

$$
\begin{array}{r}
\varepsilon=\log (1+e) \\
\sigma=S \cdot(1+e)
\end{array}
$$

Os dados são analisados considerando a aplicabilidade da equação de Hollomon:

$$
\sigma=\mathrm{K} \cdot \varepsilon^{\mathrm{n}}
$$

Após a determinação das tensões e deformações reais ( $\sigma$ e $\varepsilon$ ) é possível realizar o cálculo logarítmico dos valores de ambos, no intervalo de deformação plástica homogênea, após o início da deformação plástica generalizada do $\mathrm{CP}$ até o início de sua estricção. A partir dos dados obtidos pode ser gerada uma regressão linear, conforme:

$$
\begin{array}{r}
\log (\sigma)=\log (K)+n \cdot \log (\varepsilon) \\
Y=a+b \cdot X
\end{array}
$$

Os valores da constante plástica de resistência $(K)$ e do expoente de encruamento (n) podem ser determinados, considerando a Eq.(4) e a Eq.(5): 


$$
\begin{array}{r}
n=b \\
K=10^{a}
\end{array}
$$

\subsection{Caracterização das Condições de Trefilação}

O sistema de simulação e as condições empregadas podem ser quantificadas através dos seus parâmetros geométricos e condições superficiais. A deformação de engenharia (e) ou a redução da área $(r)$ podem ser determinadas, conhecendo-se $o$ diâmetro inicial $\left(D_{0}=3,68 \mathrm{~mm}\right)$ e o diâmetro final $\left(D_{f}=3,45 \mathrm{~mm}\right)$, através da equação:

$$
e=r=1-\left(\frac{D_{0}}{D_{f}}\right)^{2}
$$

Substituindo os resultados na Eq. (8) obtêm-se o valor de 0,1211 ou $12,11 \%$. O valor encontrado está abaixo dos $20 \%$ citados por Dieter [8] para operações comerciais, o que garante que não deveria ocorrer microfissuramento ao longo do material induzida pelo processo de trefilação. A razão de trefilação $(R)$ e a deformação (homogênea) verdadeira podem ser obtidos através de:

$$
\begin{array}{r}
\mathrm{R}=\left(\frac{A_{f}}{A_{0}}\right)=\left(\frac{D_{f}}{D_{0}}\right)^{2} \\
\mathcal{E}=\varepsilon_{\text {Hom. }}=\ln (1 / \mathrm{R})=\ln \left(\frac{A_{i}}{A_{f}}\right)=\ln \left(\frac{D_{i}}{D_{f}}\right)^{2}=2 \ln \left(\frac{D_{i}}{D_{f}}\right)
\end{array}
$$

Neste estudo, empregando-se a Eq. (9) e Eq. (10) são obtidos os seguintes valores: $\mathrm{R}=0,8789$ e $\varepsilon=0,1291$. A deformação efetiva de um processo de conformação pode ser calculada com base na equação:

$$
d \varepsilon_{e f}=\frac{\sqrt{2}}{3} \sqrt{\left(d \varepsilon_{1}-d \varepsilon_{2}\right)^{2}+\left(d \varepsilon_{2}-d \varepsilon_{3}\right)^{2}+\left(d \varepsilon_{3}-d \varepsilon_{3}\right)^{2}}
$$

A Eq. (11) pode ser simplificada, admitindo-se que o produto entre deformações possa ser desprezado, para a seguinte forma, em termos de deformação plástica total

$$
\varepsilon_{e f}=\sqrt{\frac{2}{3}\left(\varepsilon_{1}^{2}+\varepsilon_{2}^{2}+\varepsilon_{3}^{2}\right)}
$$

Considerando que na trefilação de barras ou fios cilíndricos têm-se que as deformações principais perpendiculares ao alongamento são iguais $\left(\varepsilon_{2}=\varepsilon_{3}\right)$, então torna-se possível afirmar que:

$$
\varepsilon_{\text {ef }}=\varepsilon_{\text {Tref. }}=\varepsilon_{\text {Hom }}
$$

Observação: neste caso se despreza o gradiente de deformações da parte externa para a parte interna do produto trefilado.

\subsection{Atrito e Semi-Ângulo de Trefilação}


Os valores adimensionais do coeficiente de atrito estático $(\mu)$ e dinâmico da fieira de aço em relação aos fios de cobre trefilados $\left(m_{a}\right)$ podem ser estimados através de tabelas experimentais $[9,10]$. Para o caso da simulação realizada neste trabalho, como $m_{a}>0,1$ [9] e $\mu \approx 0,06$ a 0,30 [10], então adotou-se $m_{a}=0,1$ e $\mu=0,23$. Segundo Helman e Cetlin [10] é possível determinar um valor de semi-ângulo (a) que minimize a quantidade de energia gasta no processo de trefilação, baseando-se nos valores do coeficiente de atrito e grau de redução conforme:

$$
\alpha_{\text {ót. }}=\sqrt{1,5 \cdot m_{a} \cdot \ln \left(\frac{D_{0}}{D_{f}}\right)}
$$

Com os dados da simulação ( $D_{0}=3,68 \mathrm{~mm}$; $D_{f}=3,45 \mathrm{~mm}$ e $\left.m_{a}>0,1\right)$, obtêm-se $\alpha$ ót. $=$ 0,09839 rad. ou $\alpha$ ót. $=5,637^{\circ}$. Esse valor obedece aos valores estipulados por Dieter [8] que sugere semi-ângulos na faixa de $6^{\circ}$ a $10^{\circ}$ e os valores de Button [11] que sugerem semi-ângulos de $5^{\circ}$ a $12^{\circ}$.

\subsection{Parâmetros para Análise Mecânica}

Para a determinação das cargas e deformações atuantes são empregadas equações disponíveis na literatura [5-11], deduzidas analiticamente para o processo de trefilação. Em muitas destas equações é empregado a tensão média de escoamento $(\bar{Y})$, obtida a partir da equação de Hollomon (Eq. 3) [5, 10]:

$$
\bar{Y}=\frac{K \cdot\left(\varepsilon_{e f}\right)^{n}}{(n+1)}
$$

Outro parâmetro muito empregado é o termo B que internaliza em diversas equações o efeito do atrito e da deformação redundante $[5,10,11]$ :

$$
\mathrm{B}=\mu \cdot \operatorname{cotag}(\alpha)
$$

No caso deste estudo o parâmetro $B$ pode ser calculado pelos dados do item 2.1 ( $\alpha$ $=7,5^{\circ}=0,1309$ rad.) e do item $2 \cdot 4(\mu>0,30)$ oferecendo, via Eq. (16): B > 2,2787.

É possível empregar o parâmetro de Rowe $(\phi)[5,10]$ para quantificar a relação entre a deformação redundante $\left(\varepsilon_{\text {Red. }}\right)$, associada com os fluxos metálicos na matriz, com a deformação homogênea $\left(\varepsilon_{\text {Hom. }}\right)$, calculada diretamente pelas alterações dimensionais no material conformado, Eq. (10). O parâmetro de Rowe $(\phi)$ e a deformação redundante ( $\left.\varepsilon_{\text {Red. }}\right)$ são definidos como $[5,10,11]$ :

$$
\begin{array}{r}
\phi=\frac{\left(\varepsilon_{\text {Hom. }}+\varepsilon_{\text {Red. }}\right)}{\varepsilon_{\text {Hom. }}}=\frac{\varepsilon^{*}}{\varepsilon} \\
\varepsilon_{\text {Red. }}=(\phi-1) \cdot \varepsilon_{\text {Hom. }} .
\end{array}
$$

A determinação analítica do parâmetro de Rowe pode ser feita de várias formas. Neste trabalho foram empregadas as equações devidas à Dieter [8]; Helman e Cetlin [10] e Button [11], respectivamente $\left(\phi_{1}, \phi_{2}, \phi_{3}\right)$ :

$$
\begin{array}{r}
\phi_{1} \approx 0,8+\frac{\Delta}{4,4} \quad \therefore \quad \Delta=\frac{\alpha}{r} \cdot[1+\sqrt{1-r}]^{2} \\
\phi_{2} \approx 0,88+0,12 \frac{h}{L} \quad \therefore \quad h=\frac{\left(D_{0}+D_{f}\right)}{2} \quad \therefore \quad L=\frac{\left(D_{i}-D_{f}\right)}{2 \cdot \operatorname{sen} \alpha}
\end{array}
$$




$$
\phi_{3} \approx 0,88+0,78 \frac{A_{S}}{M} \quad \therefore \quad \frac{A_{S}}{M}=\frac{D_{i}+D_{f}}{D_{i}-D_{f}}\left(\frac{1-\cos \alpha}{2 \operatorname{sen} \alpha}\right)
$$

Considerando os dados deste estudo, obtêm-se: $\phi_{1}=1,722 ; \phi_{2}=1,366 ; \phi_{3}=1,672$.

A estimativa da eficiência do processo $(\eta)$, é empregada para corrigir os valores de tensão ou força, obtidos diretamente dos equacionamentos oriundos do método da deformação uniforme [5, 10]. É um parâmetro praticamente empírico de determinação muitas vezes experimental e que varia de acordo com o processo de conformação mecânica:

$$
\eta=\frac{\sigma_{C A L C U L A D A}}{\sigma_{\text {REAL }}}
$$

Os valores da eficiência do processo serão determinados com o uso das equações do item 2.6 e com os resultados de carga obtidos diretamente da máquina de tração.

\subsection{Descrição das Cargas e Deformações Atuantes}

A tensão de conformação por trefilação ( $\sigma$ Tref) pode ser obtida diretamente pelo método da deformação homogênea $[5,10,11]$ :

$$
\sigma=\overline{\mathrm{Y}} \cdot \ln \left(\frac{A_{0}}{A_{f}}\right)=\overline{\mathrm{Y}} \cdot \ln \left(\frac{1}{1-\mathrm{r}}\right)
$$

Através do método dos blocos [3, 5, 9-11] é possível deduzir várias equações, das quais as seguintes valem para a trefilação:

$$
\begin{array}{r}
\sigma=\overline{\mathrm{Y}} \cdot(1+B) \cdot \ln \left(\frac{A_{0}}{A_{f}}\right) \\
\sigma=\overline{\mathrm{Y}} \cdot \frac{(1+\mathrm{B})}{\mathrm{B}} \cdot\left[1-\left(\frac{A_{f}}{A_{0}}\right)^{B}\right] \\
\sigma=\overline{\mathrm{Y}} \cdot\left[\left(1+\frac{2 \mu}{\operatorname{sen}(2 \alpha)}\right) \cdot \ln \left(\frac{A_{0}}{A_{f}}\right)+\frac{2}{3} \tan (\alpha)\right]
\end{array}
$$

As equações (24) e (25) não contemplam a deformação redundante $\left(\varepsilon_{\text {Red. }}\right)$, por isso é necessário substituir a deformação empregada nestas equações pela deformação redundante $\left(\mathcal{E}_{\text {Red. }}\right)$, calculada pela $\mathrm{Eq}$. (18). Além disso, a relação entre a área inicial $\left(A_{0}\right)$ e a área final $\left(A_{f}\right)$ deve ser corrigida pelo parâmetro de Rowe $(\phi)$, conforme:

$$
\begin{array}{r}
\varepsilon_{\text {Hom. }}=\varepsilon=\ln \left(\frac{A_{0}}{A_{f}}\right) \\
\left(\frac{A_{0}}{A_{f}}\right)^{*}=\exp \left(\varepsilon_{\text {Hom. }}+\varepsilon_{\text {Red. }}\right)=\exp \left(\varepsilon^{*}\right)=\exp (\phi \cdot \varepsilon)
\end{array}
$$

Finalmente é possível empregar equações desenvolvidas pelo método do limite superior, que oferecer valores de tensão de conformação tendenciosamente mais elevados dos que os valores reais [2, 5, 10, 11]. A vantagem é poder cercar os valores corretos da força de conformação e assim obter uma maior precisão nas previsões. A equação mais conhecida e aplicada para a trefilação é $[5,11]$ :

$$
\sigma=\overline{\mathrm{Y}} \cdot 2 \mathrm{f}(\alpha) \ln \left(\frac{D_{0}}{D_{f}}\right)+\frac{2 \bar{Y}}{\sqrt{3}}\left[\frac{\alpha}{\operatorname{sen}^{2}(\alpha)}-\operatorname{cotg}(\alpha)+m_{a} \cdot \operatorname{cotg}(\alpha) \cdot \ln \left(\frac{A_{0}}{A_{f}}\right)+m_{a} \frac{2 L}{D_{f}}\right]
$$


Onde $\mathrm{f}(\alpha)$ é uma função geométrica dada em função do semi-ângulo da fieira e que neste caso vale 1,0003589 para $\alpha=7,5^{\circ}$ (ou 0,1309 rad.). Usando estes mesmos parâmetros da Eq. (29) é possível analisar a trefilação, também empregando as energias gastas tanto no início, no final e a resultante do processo, de forma a obter uma relação de redução máxima $\left(\mathrm{D}_{0} / \mathrm{D}_{\mathrm{f}}\right)$ máx que o material trefilado pode alcançar, que neste trabalho foi de 1,37 (menor do que o valor de $D_{0} / D_{f}=1,07$, empregado nas simulações), conforme a seguinte equação:

$$
\left(\frac{D_{0}}{D_{f}}\right) \text { máx }=\exp \left(\frac{1-\left(\frac{2}{\sqrt{3}}\right) \cdot\left(\left(\frac{\alpha}{\operatorname{sen}^{2} \alpha}\right)-(\operatorname{ctg} \alpha)\right)}{2 \cdot\left(\mathrm{f}(\alpha)+\left(\frac{m_{a}}{\sqrt{3} \cdot \operatorname{ctg} \alpha}\right)\right)}\right)
$$

\section{RESULTADOS E DISCUSSÃO}

A partir dos dados coletados no ensaio para cada corpo de prova, foi possível gerar gráficos Força $x$ Deslocamento, conforme ilustrado na Figura 2.a. Nesse gráfico estão exemplificados os resultados de quatro experiências de trefilação com condições variadas de lubrificação e velocidade (vide Tab. 1). Como os resultados flutuaram muito, tornou-se necessária uma análise estatística com estes dados de modo a filtrar resultados extremos. Esta análise foi feita através de gráficos de probabilidade, geradas através do software MiniTab ${ }^{\circledR}$, como os mostrados na Figura 2.b.

Neste caso, foram seguidamente suprimidos os registros de força abaixo de $5 \%$ e acima de $95 \%$ dos resultados registrados até uma melhoria do comportamento dos dados, que apesar desta redução, ainda são de uma grande quantidade (de 432 a 1147 valores de força medidos e aproveitados). Os resultados desta análise estão apresentados nas Figuras 2.c e 2.d. Mesmo com a redução na dispersão dos resultados das forças de trefilação, por exemplo na Tref. 3 - CP4, o desvio padrão caiu de $88,7 \mathrm{~N}$ para $52,11 \mathrm{~N}$ (variação de $-41 \%$ ); as médias obtidas pouco mudaram, por exemplo na Tref. 5 - CP7: FMéd. de 697,4 N para 710,9 N (variação de +1,9\%).

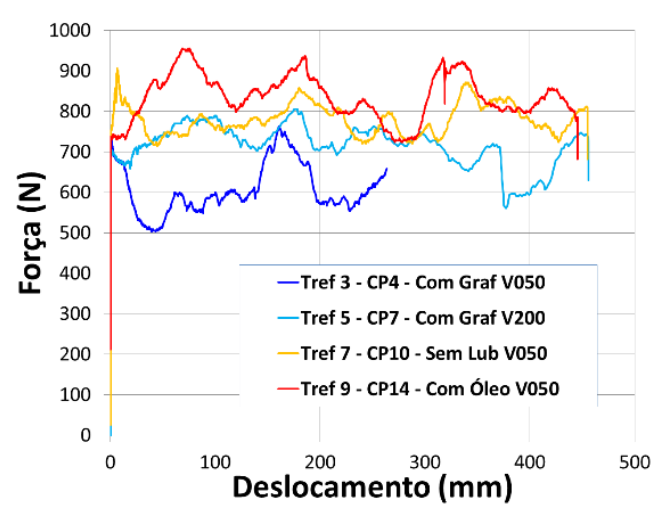

(a)

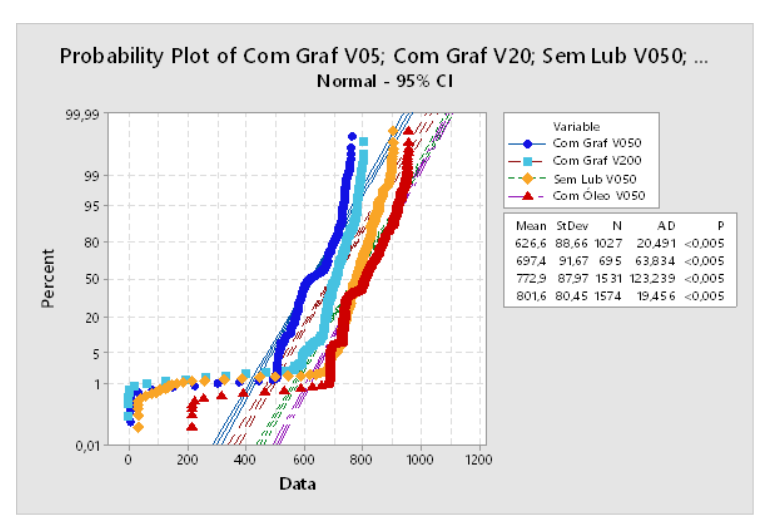

(b) 


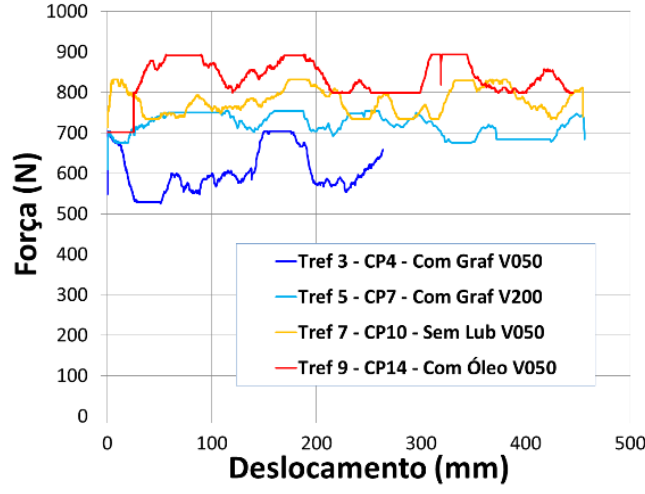

(c)

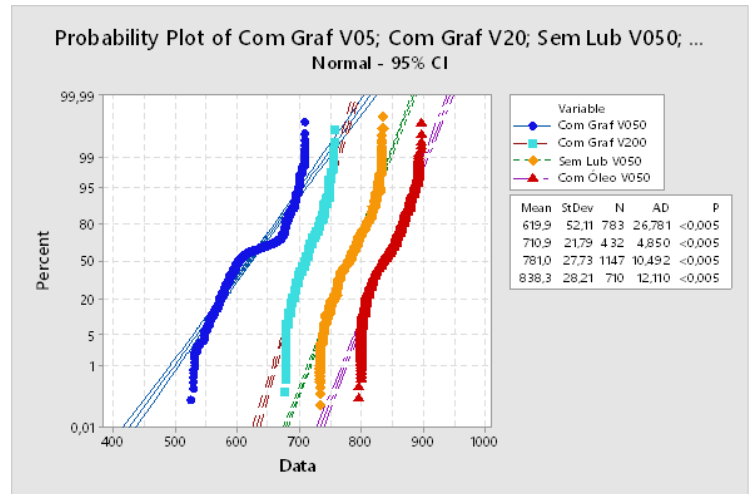

(d)

Figura 2. Exemplos de dados obtidos durante a simulação da trefilação: (a) curvas Força $x$

Deslocamento e (b) curvas de probabilidade de força. Dados filtrados estatisticamente: (c) curvas Força x Deslocamento e (d) curvas de probabilidade de força.

Os dados de força de trefilação obtidos nas simulações, representados pela força observada com $50 \%$ de probabilidade (moda) no processo, estão apresentados na Tabela 2. Não estão disponíveis os dados de trefilação sem lubrificação a 50 $\mathrm{mm} / \mathrm{min}$. devido à ruptura constante dos fios sendo trefilados nesta condição.

Tabela 2. Força de trefilação $\left(F_{50 \%}\right)$ em função das condições de trefilação.

\begin{tabular}{cccc}
\hline $\begin{array}{c}\text { Condição de } \\
\text { lubrificação }\end{array}$ & $\begin{array}{c}\text { Velocidade } \\
(\mathbf{m m} / \mathbf{m i n} .)\end{array}$ & $\begin{array}{c}\text { Força de trefilação } \\
-\mathbf{F}_{50 \%}(\mathbf{N})\end{array}$ & $\begin{array}{c}\text { Tensão de trefilação } \\
-\sigma_{\text {Real }}(\mathbf{M P a})\end{array}$ \\
\hline Óleo & 50 & $828^{(*)}$ & 88,57 \\
\hline \multirow{2}{*}{ Sem lubrificação } & 50 & n.d. $^{(*)}$ & n.d..$^{(*)}$ \\
\hline \multirow{2}{*}{ Grafite em pó } & 200 & 785 & 83,97 \\
\cline { 2 - 4 } & 200 & 683 & 73,06 \\
\hline
\end{tabular}

$\left(^{*}\right)$ Neste caso a quantidade de testes obtidos foram poucos $(\mathrm{N}<3)$, irregulares e assim desprezados.

O gráfico da Figura 3.a ilustra algumas das curvas de tração em termos de tensão versus deformação de engenharia $(S \times e)$ para arames antes e após a trefilação. Estes dados foram analisados empregando-se as Equações (1) a (7) para serem obtidos os valores das constantes da Equação de Hollomon - Eq. (3) a partir de regressões lineares - Eq. (4) dos dados processados das curvas de tração. A Figura 3.b ilustra as retas geradas pelas curvas mostradas no gráfico da Figura 3.a.

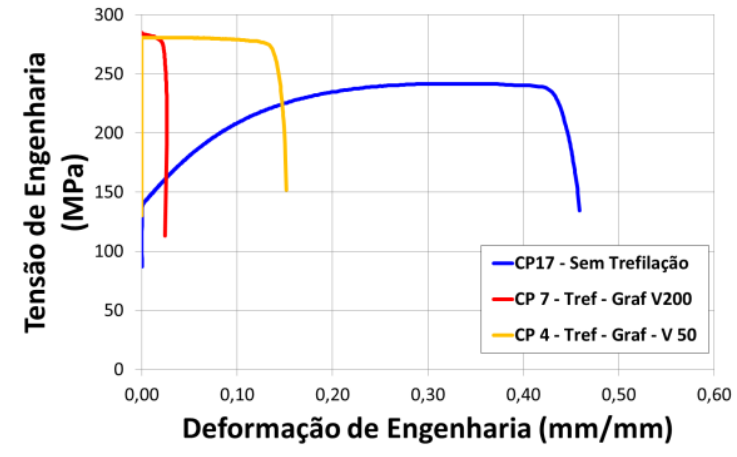

(a)

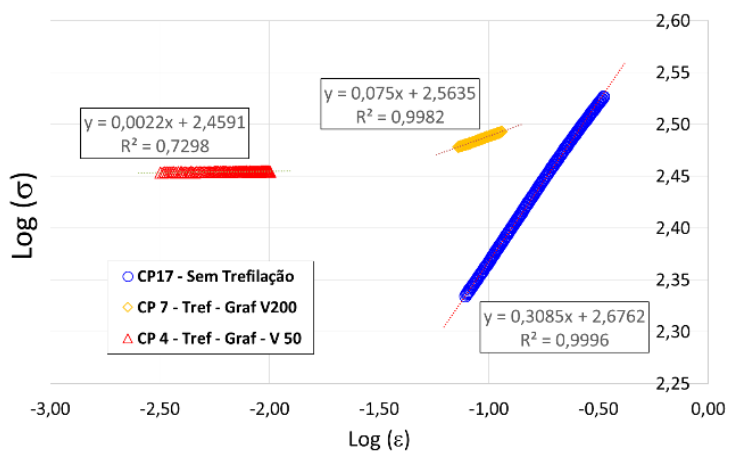

(b) 
Figura 3. Alguns resultados de caracterização mecânica dos arames de cobre antes e depois da trefilação: (a) curvas tensão versus deformação de engenharia $(S \times e)$ e $(b)$ regressões lineares $\log (\sigma) \times \log (\varepsilon)$ para determinação dos parâmetros da equação de Hollomon (K e n) - Eq. (3).

As três condições representadas pelos gráficos da Figura 3 oferecem os seguintes resultados, em termos de parâmetros da equação de Hollomon (K e n) - Eq. (3):

- $\mathrm{CP} 17$ - Sem trefilação - $\mathrm{K}=474 \mathrm{MPa}$ e $\mathrm{n}=0,3085$;

- CP 7 - Trefilado com lubrificação de grafite a $200 \mathrm{~mm} / \mathrm{min}$. $-\mathrm{K}=366 \mathrm{MPa}$ e $\mathrm{n}=0,075$;

- CP 4 - Trefilado com lubrificação de grafite a $50 \mathrm{~mm} / \mathrm{min}$. $-\mathrm{K}=288 \mathrm{MPa}$ e $\mathrm{n}=0,0022$.

É possível perceber, pelos dados obtidos, como o encruamento advindo do processo de trefilação executado reduz a capacidade de encruamento posterior do material. Essa característica é evidenciada pela diminuição do expoente de encruamento (n) e pela diminuição constante plástica de resistência $(K)$, que descreve o nível de resistência mecânica final que poderia ser obtido após encruamento unitário $(\varepsilon=1)$. Neste último caso, o CP7 apresenta um limite de escoamento $\left(S_{L E}\right)$ praticamente igual ao valor da constante plástica de resistência $(K)$ indicando que este fio está no seu limite da capacidade de deformação plástica. Considerando que todos os fios foram deformados com o mesmo grau de deformação homogênea, conforme definida pela Eq. (10) e Eq. (13), pode-se inferir que o processo de trefilação introduziu condições de encruamento bem diferentes nestas duas amostras.

Os dados obtidos nas simulações foram empregados nas equações de caracterização das condições do processo de conformação - Eq. (8) a Eq. (22), e nas equações deduzidas pelos métodos analíticos - Eq. (23) a Eq. (29). Os parâmetros básicos do processo estão apresentados na Tabela 3, a maioria foi definida pela fieira empregada (Fig. 1.C), sendo que os demais foram calculados conforme as equações indicadas.

Tabela 3. Condições empregadas na simulação da trefilação.

\begin{tabular}{ccccccccc}
\hline Do $(\mathbf{m m})$ & $\mathbf{D f}(\mathbf{m m})$ & $\mathbf{2} \alpha_{\text {Real }}$ & $\boldsymbol{\alpha}_{\text {Real }}$ & $\mathbf{r}$ (ad.) & $\mathbf{A}_{\mathbf{0}}\left(\mathbf{m m}^{\mathbf{2}}\right)$ & $\mathbf{A}_{\mathbf{f}}\left(\mathbf{m m}^{\mathbf{2}}\right)$ & $\mathbf{R}$ & $\boldsymbol{\varepsilon}_{\text {Hom. }}$ \\
\hline 3,68 & 3,45 & $\begin{array}{c}15^{\circ} \\
\text { ou } 0,2618 \\
\text { rad. }\end{array}$ & $\begin{array}{c}7,5^{\circ} \\
\text { ou } 0,1309 \\
\text { rad. }\end{array}$ & $\begin{array}{c}0,1211 \\
\text { ou } 12,11 \% \\
\text { Eq. (8) }\end{array}$ & 10,63 & 9,348 & 0,8789 & 0,1291 \\
& & & & & Eq. (9) & Eq. (10) \\
\hline
\end{tabular}

Empregando os dados do processo é possível determinar parâmetros básicos para a análise mecânica do processo simulado. Estes dados, obtidos das equações apresentadas neste trabalho ou de referências bibliográficas, para o caso específico dos coeficientes de atrito, estão apresentados na Tabela 4. Estes parâmetros serão empregados nas equações de determinação da tensão de trefilação.

Tabela 4. Parâmetros básicos para a análise mecânica da simulação da trefilação.

\begin{tabular}{ccccccccc}
\hline $\mathbf{K}(\mathbf{M P a})$ & $\mathbf{n}$ & $\boldsymbol{\mu}$ & $\mathbf{m}_{\mathbf{a}}$ & $\overline{\mathbf{Y}}$ & $\mathbf{B}$ & $\boldsymbol{\phi} 1$ & $\mathbf{\phi} 2$ & $\boldsymbol{\phi} \mathbf{3}$ \\
\hline 473,3 & 0,3073 & 0,30 & 0,10 & 193,0 & 2,279 & 1,722 & 1,366 & 1,672 \\
\hline Eq. (7) & Eq. (6) & {$[6,9,10]$} & Eq. (15) & Eq. (16) & Eq. (19) & Eq. (20) & Eq. (21) \\
\hline
\end{tabular}

Empregando os dados das Tabelas 2 (forças de conformação), 3 (condições) e 4 (parâmetros básicos) é possível correlacionar estes valores com as equações da literatura [2, 5, 8-11] para obter as condições de trefilação apresentadas na Tabela 5 .

Tabela 5. Parâmetros básicos para a análise mecânica da simulação da trefilação. 


\begin{tabular}{|c|c|c|c|c|c|c|c|c|c|c|c|c|}
\hline \multirow[b]{2}{*}{$\sigma_{\text {Real }}$} & \multicolumn{3}{|c|}{$\begin{array}{l}\text { Tref. com óleo a } \\
50 \mathrm{~mm} / \mathrm{min} \text {. }\end{array}$} & \multicolumn{3}{|c|}{$\begin{array}{l}\text { Tref. sem lubrif. a } \\
200 \mathrm{~mm} / \mathrm{min} \text {. }\end{array}$} & \multicolumn{3}{|c|}{$\begin{array}{l}\text { Tref. com grafite a } \\
200 \mathrm{~mm} / \mathrm{min} .\end{array}$} & \multicolumn{3}{|c|}{$\begin{array}{l}\text { Tref. com grafite a } \\
50 \mathrm{~mm} / \mathrm{min} \text {. }\end{array}$} \\
\hline & 88,57 & 88,57 & 88,57 & 83,97 & 83,97 & 83,97 & 73,06 & 73,06 & 73,06 & 70,39 & 70,39 & 70,39 \\
\hline$\sigma_{\text {Hom. }}$ & 24,91 & 24,91 & 24,91 & 24,91 & 24,91 & 24,91 & 24,91 & 24,91 & 24,91 & 24,91 & 24,91 & 24,91 \\
\hline$\eta$ & $28 \%$ & $28 \%$ & $28 \%$ & $28 \%$ & $28 \%$ & $28 \%$ & $28 \%$ & $28 \%$ & $28 \%$ & $28 \%$ & $28 \%$ & $28 \%$ \\
\hline$\varepsilon_{\text {Hom. }}$ & 0,129 & 0,129 & 0,129 & 0,129 & 0,129 & 0,129 & 0,129 & 0,129 & 0,129 & 0,129 & 0,129 & 0,129 \\
\hline$\phi$ & 1,72 & 1,67 & 1,37 & 1,12 & r & 1,37 & 1,12 & 1,67 & 1,37 & 1,12 & 1,67 & 1,37 \\
\hline$\varepsilon_{\text {Red. }}$ & 0,093 & 0,087 & 0,047 & 0,093 & 0,087 & 0,047 & 0,093 & 0,087 & 0,047 & 0,093 & 0,087 & 0,047 \\
\hline$\overline{\mathbf{Y}}^{*}$ & 228,1 & 226,0 & 212,4 & 228,1 & 226,0 & 212,4 & 228,1 & 226,0 & 212,4 & 228,1 & 226,0 & 212,4 \\
\hline$\varepsilon_{\text {Atri }}$ & 0,118 & 0,125 & 0,164 & 0,105 & 0,111 & 0,151 & 0,072 & 0,078 & 0,118 & 0,063 & 0,070 & 0,109 \\
\hline$\overline{\mathbf{Y}} * *$ & 260,0 & 260,0 & 260,0 & 256,8 & 256,8 & 256,8 & 248,5 & 248,5 & 248,5 & 246,4 & 246,4 & 246,4 \\
\hline$\sigma_{\text {Hom }} * *$ & 88,6 & 88,6 & 88,6 & 84,0 & 84,0 & 84,0 & 73,1 & 73,1 & 73,1 & 70,4 & 70,4 & 70,4 \\
\hline$\eta * *$ & $100 \%$ & $100 \%$ & $100 \%$ & $100 \%$ & $100 \%$ & $100 \%$ & $100 \%$ & $100 \%$ & $100 \%$ & $100 \%$ & $100 \%$ & $100 \%$ \\
\hline$\sigma_{\text {Bloc. }} \mathrm{Eq}(24)$ & 139,2 & 134,0 & 102,9 & 139,2 & 134,0 & 102,9 & 139,2 & 134,0 & 102,9 & 139,2 & 134,0 & 102,9 \\
\hline$\sigma_{\text {Bloc. }} \mathrm{Eq}(25)$ & 115,4 & 111,6 & 88,5 & 115,4 & 111,6 & 88,5 & 115,4 & 111,6 & 88,5 & 115,4 & 111,6 & 88,5 \\
\hline$\sigma_{\text {Bloc. }} \mathrm{Eq}(26)$ & 160,8 & 155,3 & 122,6 & 160,8 & 155,3 & 122,6 & 160,8 & 155,3 & 122,6 & 160,8 & 155,3 & 122,6 \\
\hline$\mu \quad E q(25)$ & & 0,230 & & & 0,206 & & & 0,152 & & & 0,140 & \\
\hline
\end{tabular}

$\mathrm{Na}$ Tabela 5 todos os valores das tensões obtidas pelo método dos blocos foram corrigidos pelos parâmetros de Rowe $\left(\phi_{1}, \phi_{2}, \phi_{3}\right)$ de forma que:

- a deformação empregada é a soma das deformações homogênea e redundante $\left(\varepsilon_{\text {Hom. }}+\varepsilon_{\text {Red. }}\right)$, lembrando que o atrito é introduzido diretamente pelo uso do coeficiente de atrito estático $(\mu)$ nestas equações;

- no caso da Eq. (25) empregou-se a relação de áreas $-\left(A_{0} / A_{f}\right)^{*}=\exp \left(\varepsilon_{\text {Hom. }}+\varepsilon_{\text {Red. }}\right)$ corrigida pela Eq. (28).

Empregando essas condições, as equações devidas ao método dos Blocos deveriam obter os valores da tensão real. Observando-se os dados da Tabela 5, nota-se que os valores obtidos pelo uso da Eq. (25), empregando o parâmetro de Rowe $\left(\phi_{2}=1,37\right)$ devido a Helman e Cetlin [10], ofereceram os melhores resultados, com um erro médio de $13 \%$, quando comparado os resultados obtidos com a tensão real medida.

As deformações por atrito $\left(\varepsilon_{\text {Atrito }}\right)$ foram obtidas pelo seguinte procedimento:

1. acrescenta-se aos valores de deformação homogênea ( $\left.\varepsilon_{\text {Hom. }}\right)$, originalmente calculados pela Eq. (10), o valor da deformação redundante $\left(\varepsilon_{\text {Red. }}\right)$, estimado pela Eq.

(18) e um valor de deformação por atrito $\left(\varepsilon_{\text {Atrito }}\right)$ inicialmente estimada em 0,1 ;

2. emprega-se a nova deformação obtida $\left(\varepsilon_{\text {Hom. }}+\varepsilon_{\text {Red. }}+\varepsilon_{\text {Atrito }}\right)$ nas equações da tensão média de deformação $(\overline{\mathbf{Y}})$ - Eq (15) e na tensão obtida pelo método da deformação homogênea $\left(\sigma_{\text {Hom. }}\right)$ - Eq. (23);

3. Determina-se o valor exato da deformação por atrito $\left(\varepsilon_{\text {Atrito }}\right)$ de forma que a eficácia do processo $(\eta * *)$ torne-se $100 \%$.

Adotando-se o parâmetro de Rowe $\left(\phi_{2}=1,37\right)$ devido a Helman e Cetlin [10], conforme verificado anteriormente, concluiu-se que a quantificação do atrito está coerente com as condições de processo. O atrito aumenta com as alterações das condições de lubrificação e velocidades. Neste caso em particular, percebeu-se a pouca eficácia da lubrificação líquida, realizada por meio de óleo. Os dados demonstram quem houve pouca alteração das deformações por atrito entre estas duas condições e a prática mostrou que houve pouca variação nas condições de trefilação empregando-se óleo ou não empregando lubrificação nenhuma. 
Além disso, inicialmente foi considerado um valor de coeficiente de atrito estático constante e igual à $(\mu=0,23)$. Porém esta não é uma condição correta, já que houve alteração das condições de lubrificação, inclusive com a evidência não apenas da redução da tensão real ( $\sigma$ Real) como também da deformação por atrito (EAtrito). Os valores de $\mu$ foram determinados de tal forma que o seu uso na Eq. (25), que foi a mais precisa neste estudo, forneçam os resultado reais da tensão de trefilação. Os valores obtidos de $\mu$ através deste procedimento estão mostrados na Tabela 5.

\section{CONCLUSÃO}

Empregando-se os dados gerados nos ensaios de caracterização do material e os dados obtidos durante as simulações de trefilação na máquina de tração, é possível comparar matematicamente os resultados práticos obtidos com os valores teóricos calculados. Além disso, podem ser investigadas diferentes condições de trefilação para quantificar o efeito sobre o esforço médio de conformação.

Com isso, é possível empregar um recurso de acesso relativamente comum (máquina de tração) para simular e analisar o processo de trefilação de forma simples e prática. O trabalho executado demonstra o potencial de emprego desta metodologia na análise das condições de trefilação e consequentemente para melhorias do processo de trefilação, como por exemplo, na quantificação do uso de novos lubrificantes.

\section{REFERÊNCIAS}

1 MORAIS, W.A.; SANTOS, A. P. ; SANTOS, D. R. ; NASCIMENTO, M. R. V. O. . Simulação do processo de trefilação através de uma máquina de tração. In: 3o Seminário de Trefilação, 2012, São Paulo. Anais do 3ํㅗㄹ Seminário de Trefilação da ABM. São Paulo: ABM, 2012. p. 38-50.

2 BRECIANI FILHO, E.; SILVA, I. B.; BATALHA, G. F.; BUTTON, S. T. Conformação plástica dos metais; $2011,3^{\circ} \mathrm{Ed}$., Trefilação, p 48-60.

3 JUNIOR, W. B., Analise da lubrificação seca para trefilação e proposta de padronização de lubrificantes para arame de médio porte [monografia de graduação], Porto Alegre, Universidade Federal do Rio Grande do Sul, 2009.

4 MAGALHÃES, F. C; PERTENCE, A. E. M.; CAMPOS, H. B.; AQUILAR, M. T. P.; CETLIN, P. R. Evolução de defeitos superficiais em barras de cobre submetidas ao processo de trefilação. In: Associação Brasileira de Engenharia e Ciências Mecânicas 2011, $6^{\circ}$ Congresso Brasileiro de Engenharia de Fabricação, 2011, Caxias do Sul (RS), Brasil.

5 MORAIS, Willy Ank, Processos de Conformação dos Metais; 2010, Cap. 4. Métodos de Cálculo, Apostila UNISANTA. p 68-126.

6 COEFICIENTES DE ATRITO PARA ALGUNS MATERIAIS COMUNS E COMBINAÇÕES DE MATERIAIS. Caixa de Ferramenta do Engenheiro, 2007. Disponível em: <http://www.engineeringtoolbox.com/friction-coefficients-d 778.html> Acesso em:14 jun. 2016.

7 NUNES, Laerce de Paula; Kreischer, Anderson Teixeira. Introdução Metalurgia e aos Materiais Metálicos. Rio de Janeiro: Interciência,2010.

8 DIETER, George E. Metalurgia mecânica. Traduzido por . 2.ed. ed. Rio de Janeiro: Guanabara Dois, 1981, 653 p.

9 SCHAEFFER, L.; Conformação Mecânica. Imprensa Livre Editora, Porto Alegre, 1999.

10 HELMAN, H.; CETLIN, P.R.; Fundamentos da Conformação Mecânica dos Metais. Editora Guanabara Dois, 1983. 
11 BUTTON, S.T.; Curso: Trefilação. Associação Brasileira de Metalurgia e Materiais, São Paulo, 2002. 